\title{
Screening of Different $B t$ Cotton Genotypes against Yellow Mite, Polyphagotarsonemus latus (Banks) and Biochemical Basis of Resistance
}

\author{
K. A. Biradarpatil ${ }^{1^{*}}$, S. T. Prabhu ${ }^{2}$ and M. R. Ravikumar ${ }^{3}$ \\ ${ }^{1}$ Agricultural Entomology, University of Agricultural Sciences, Dharwad, Karnataka, India \\ ${ }^{2}$ Department of Agricultural Entomology, College of Agriculture Hanumanamatti, University \\ of Agricultural Sciences, Dharwad, Karnataka, India \\ ${ }^{3}$ Department of Plant Pathology, College of Agriculture Hanumanamatti, University of \\ Agricultural Sciences, Dharwad, Karnataka, India \\ *Corresponding author
}

\begin{tabular}{l} 
Ke y w o r d s \\
Cotton, Yellow \\
mite, Genotypes, \\
$\begin{array}{l}\text { Morphological } \\
\text { character, } \\
\text { Biochemical } \\
\text { character }\end{array}$ \\
\hline Article Info \\
\hline $\begin{array}{l}\text { Accepted: } \\
\text { 15 December } 2019 \\
\text { Available Online: } \\
\text { 20 January } 2020\end{array}$ \\
\hline
\end{tabular}

Keywords

Cotton, Yellow mite, Genotypes Morphological character, Biochemica characte

Accepted: 15 December 2019 20 January 2020

\section{A B S T R A C T}

Field trial was conducted for two consecutive years during kharif 2017 and 2018 at farmer`s field, Kadarmandalagi by using randomized block design (RBD). During kharif 2017, among the 20 genotypes screened indicated that lowest mean population of yellow mite and highest mean leaf hair density and trichome length were recorded in Chaitanya (MRC-7377) (0.29 yellow mite/ leaf) followed by Soumya (SRCH-55) and were superior to all the genotypes. While highest mean yellow mite population and least mean leaf hair density and trichome length were observed in Super fibre (KDCHB-407) (2.96 yellow mite/ leaf). During kharif 2018, two resistant and two susceptible genotypes were chosen based on performance during kharif 2017 for biochemical cgaracter analysis. Morphological characters like trichomes and trichome length and biochemical character phenol were recorded highest in resistant genotypes Chaitanya (MRC-7377) and Soumya (SRCH-55). While, susceptible genotypes Super fibre (KDCHB-407) and Surya (Namkot-803) were recorded lowest trichomes and trichome length and highest sugars and proteins.

\section{Introduction}

Cotton (Gossypium spp.) is the world's most important natural textile fibre crop and its seeds are good source of vegetable oil and protein meal. It is considered as king of fiber and white gold because of most superior fiber and garment qualities with highest economic returns. Indian textile industry predominantly depends on cotton to an extent of 60 per cent which contributes around four per cent to the country's gross domestic product (GDP), 14 per cent to industrial production and 13 per cent to total export earnings, providing 
employment to over 45 million people directly and 60 million people indirectly (Anonymous 2016) ${ }^{(1)}$.

Since the introduction and commercialization of $B t$ transgenic genotypes in 2002 by Genetic Engineering Appraisal Committee (GEAC) there is a drastic increase in area and production of cotton in India. Further, commercialization of Bollgard-II (2006) cottons expressing CrylAc and $C r y 2 A b$ together paved way for dominance of emerging pests in cotton. Hence, there is mosaic of different hybrids and $B t$ genes offering varied level of resistance /susceptibility to bollworms and sucking pests. Emerging pests have become significant issue in sustainability of $B t$ cottons in many countries. It is difficult to manage these pests and control failures have been experienced by the cotton growers due to repeated use of insecticides. Though control failure may be due to many factors, of which the development of resistance to insecticides is the major one (Jeya PS, Regupathy A) ${ }^{(5)}$. Hence, host plant resistance is one of the important tools in IPM to combat the pest throughout the crop stage. It is not only the non-cash input, but also does not demand any extra labour. Host plant - insect interaction is a dynamic, system and coevolutionary process, which involves development of the defense mechanisms by the plants and counter adaptations by the insects.

Defense mechanisms involve either morphological barriers or elaborative array of phytochemicals, which act as repellents, phagodeterrents and oviposition deterrents, thus exhibiting resistance. Cotton phenols and tannins were also found negatively correlated with sucking pest population densities (Butter et $a$ l.,) (2). It was felt necessary to screen popular Bt cotton hybrids which grown by farmers in Karnataka.

\section{Materials and Methods}

Screening of $B t$ cotton genotypes against yellow mite, $P$. latus

Twenty $B t$ cotton genotypes were screened against yellow mite incidence in a field trial in randomized block design (RBD) laid out at farmer's field, Kadarmandalagi during kharif 2017. Each genotype was sown in two rows of six meters at spacing of $90 \mathrm{~cm} \times 60 \mathrm{~cm}$ and was replicated twice. The recommended package of practices was followed to raise the crop except plant protection measures. Number of yellow mite per leaf and leaf curl index at different stages of crop and morphological characters like leaf thickness, leaf hair density, trichome length and yield characters were recorded. Morphological characters were correlated with population of yellow mite by using SPSS software.

\section{Biochemical observations}

Out of twenty genotypes screened during kharif 2017, two resistant and two susceptible genotypes were chosen based on morphological characters and leaf curl index and those selected genotypes were grown in kharif 2018 season for biochemical analysis. Each genotype was sown in two rows of six meters at spacing of $90 \mathrm{~cm} \times 60 \mathrm{~cm}$ and was replicated five times. The quantity of total chlorophyll, protein, sugar and phenol were estimated from the leaf samples at 60 days after sowing along with biophysical characters and were correlated with population of yellow mite by using SPSS software.

Total chlorophyll - by Hiscox and Israeistam (5) (1979) method

Protein - by Lowry et al., ${ }^{(7)}$ (1951)

method

Sugars - by Nelson Somogyi's method, 
Nelson ${ }^{(8)}$ (1944)

Phenols - by Sadasivam and Manikam (12) 1992 method

\section{Results and Discussion}

\section{Screening of different $B t$ cotton genotypes against yellow mite during kharif 2017}

The results of the experiment conducted to evaluate the reaction of $20 \mathrm{Bt}$ cotton hybrids against yellow mite along with morphological, biochemical and yield parameters of the genotypes are presented hereunder.

Twenty genotypes were screened during the year kharif 2017 against cotton yellow mite (Table 3). The population of yellow mite in different $B t$ cotton genotypes was recorded from 30 DAS up to 90 DAS at 15 days intervals. At 30 days, Chaitanya (MRC-7377) recorded least incidence of yellow mite $(0.32$ yellow mite/ leaf) and the next best genotypes in order were Soumya (SRCH-55) (0.49 yellow mite/ leaf) and Ajeet - 199 (0.85 yellow mite/ leaf) which recorded significantly less yellow mite population and were on par with each other.

However, more number of yellow mite population recorded on Super fibre (KDCHB407) (3.59 yellow mite/ leaf), Surya (Namkot803) (3.28 yellow mite/ leaf), Minerva (SP911B2) (2.73 yellow mite/ leaf) and MRC-7351 (2.47 yellow mite/ leaf) and were statistically on par with each other. However, peak yellow mite population was observed at 45 days after sowing with highest population of 4.78 yellow mite per leaf in Super fibre (KDCHB-407) followed by Surya (Namkot803) (4.62 yellow mite/ leaf), Minerva (SP911B2) (3.89 yellow mite/ leaf) and MRC-7351 (3.79 yellow mite/ leaf). The least population of yellow mite was recorded on Chaitanya (MRC-7377) (0.78 yellow mite/ leaf), Soumya (SRCH-55) (0.93 yellow mite/ leaf) and Ajeet - 199 (1.38 yellow mite/ leaf) which were significantly superior among other genotypes. The trend was similar at 60 days after sowing but the population of yellow mite decreased gradually up to 90 days after sowing. Chaitanya (MRC-7377) recorded least population of yellow mite by recording $0.21,0.12$ and 0.00 yellow mite per leaf at 60, 75 and 90 DAS respectively followed by Soumya (SRCH-55) (0.28 yellow mite/ leaf, 0.19 yellow mite/ leaf and 0.00 yellow mite/ leaf) and Ajeet - 199 (0.40 yellow mite/ leaf, 0.26 yellow mite/ leaf and 0.00 yellow mite/ leaf) and were significantly superior over all the genotypes while highest population was recorded in Super fibre as $3.17,2.66,0.58$ yellow mite per leaf at 60,75 and 90 DAS respectively.

However, least mean yellow mite population was recorded in Chaitanya (MRC-7377) (0.29 yellow mite/ leaf) which was followed by Soumya (SRCH-55) (0.38 yellow mite/ leaf) and Ajeet - 199 (0.58 yellow mite/ leaf) which were on par with each other and significantly superior to rest of the genotypes. The highest yellow mite population was observed in Super fibre (KDCHB-407) (2.96 yellow mite/ leaf) followed by Surya (Namkot-803) (2.77 yellow mite/ leaf), Minerva (SP911B2) (2.29 yellow mite/ leaf) and MRC-7351 (2.18 yellow mite/ leaf) (Table 3 ).

With respect to mean leaf curl index, Super fibre (KDCHB-407) recorded the highest leaf curl index (2.39 LCI) followed by Surya (Namkot-803) (2.30 LCI), Minerva (SP911B2) (2.07 LCI) and MRC-7351 (2.01 LCI) which were at par with each other. On the contrary, least leaf curl index was observed in Chaitanya (MRC-7377) (0.53 LCI), Soumya (SRCH-55) (0.58 LCI) and Ajeet - 199 (0.66 LCI) which showed significantly superiority over other genotypes (Table 3). 
Morphological character of different $B t$ cotton genotypes for their correlation with mite during kharif 2017

Trichomes influence oviposition and feeding in a wide range of insects and other herbivores. Leaf thickness also influences on feeding of many sucking pests but on contrary, some may evolve physiological or behavioral traits that allow them to cope with structural plant defense.

\section{Leaf thickness (mm)}

Ajeet - 155 recorded non significantly higher leaf thickness of $0.36 \mathrm{~mm}$ followed by Bioseed yuva (0.34), MRC - $7383(0.34 \mathrm{~mm})$ and Champion $(0.33 \mathrm{~mm})$ and were on par with each other. However, lowest leaf thickness recorded in Miracle $(0.25 \mathrm{~mm})$ followed by Soumya (SRCH-55) $(0.26 \mathrm{~mm})$, Nawab (0.26 mm), Minerva (SP911B2) (0.27 $\mathrm{mm})$ and Super fibre (KDCHB-407) (0.27 $\mathrm{mm}$ ) (Table 4)

\section{Leaf hair density (No. / $0.5 \mathrm{~cm}^{2}$ )}

Significantly higher numbers of leaf hairs were recorded in genotype Chaitanya (MRC7377) (144.89/0.5 $\left.\mathrm{cm}^{2}\right)$, Soumya (SRCH-55) $\left(138.17 / 0.5 \mathrm{~cm}^{2}\right)$ and Ajeet - $199(131.06 / 0.5$ $\mathrm{cm}^{2}$ ) whereas Super fibre (KDCHB-407) $\left(12.93 / 0.5 \mathrm{~cm}^{2}\right)$, Surya (Namkot-803) $(19.63$ $\left./ 0.5 \mathrm{~cm}^{2}\right)$, Minerva (SP911B2) (30.19/0.5 $\left.\mathrm{cm}^{2}\right)$ and MRC-7351 (47.31 $\left./ 0.5 \quad \mathrm{~cm}^{2}\right)$ recorded significantly least trichomes. However, the genotypes viz., Flux (PCH-888) (125.50/0.5 $\left.\mathrm{cm}^{2}\right)$, Gajab $\left(117.44 / 0.5 \mathrm{~cm}^{2}\right)$, Bioseed yuva $\left(112.17 / 0.5 \mathrm{~cm}^{2}\right)$ and Ajeet $155\left(109.61 / 0.5 \mathrm{~cm}^{2}\right)$ had moderate trichome density (Table 4).

\section{Trichome length (mm)}

The longest trichomes was observed in Chaitanya (MRC-7377) $(0.73 \mathrm{~mm})$ and was on par with Soumya (SRCH-55) $(0.61 \mathrm{~mm})$ and Flux (PCH-888) $(0.49 \mathrm{~mm})$ which were significantly superior to all the genotypes followed by Ajeet - 199 (0.40 mm), Gajab $(0.38 \mathrm{~mm})$, Ajeet - $155(0.37 \mathrm{~mm})$ and Bioseed yuva $(0.35 \mathrm{~mm})$ while, comparatively smaller trichomes were noticed in Super fibre (KDCHB-407) $(0.12 \mathrm{~mm})$, Surya (Namkot803) (0.19 mm), Minerva (SP911B2) (0.21 $\mathrm{mm})$ and MRC-7351 (0.29 mm) (Table 4).

\section{Correlation studies}

The correlation coefficient was worked out on the number of yellow mite as a dependent variable and leaf morphological characters as independent variables. The correlation coefficients indicated that there was no significant correlation between the mean number of yellow mite with leaf thickness whereas leaf hair density $(-0.974 * *)$ and trichome length $\left(-0.810^{* *}\right)$ showed highly significant and negative correlation on mean number of yellow mite (Table 4).

Yield and yield parameters of different $B t$ cotton genotypes screened for yellow mite during kharif 2017

\section{Good Opened Bolls (GOB'S)}

Data on GOB's recorded during kharif 2017 revealed that Chaitanya (MRC-7377) (50.07 bolls/plant) recorded significantly highest number of GOB's followed by Soumya (SRCH-55) (45.33 bolls/ plant), Ajeet - 199 (44.14 bolls/ plant), Flux (PCH-888) (42.42 bolls/ plant) and Gajab (40.67 bolls/ plant). While, least number of GOB's was recorded in Super fibre (KDCHB-407) (17.53 bolls/ plant) followed by Surya (Namkot-803 bolls/ plant) (20.54 bolls/ plant), Minerva (SP911B2) (22.55 bolls/ plant) and MRC7351 (23.75 bolls/ plant) which were significantly on par each other (Table 5). 


\section{Bad Opened Bolls (BOB'S)}

Data on BOB's during kharif 2017 presented in table 15 revealed that Chaitanya (MRC7377) was significantly superior in recording least BOB's (6.13 bolls/ plant) and was on par with Soumya (SRCH-55) (7.11 bolls/ plant) and Ajeet - 199 (7.58 bolls/ plant). While Super fibre (KDCHB-407) (12.11 bolls/ plant) recorded significantly more number of BOB's and was on par with Surya (Namkot803) (11.89 bolls/ plant), Minerva (SP911B2) (11.71 bolls/ plant) and MRC-7351 (11.43 bolls/ plant) (Table 5).

\section{Seed cotton yield (q/ha)}

Data on seed cotton yield during kharif 2017 revealed that Chaitanya (MRC-7377) was significantly superior in recording highest yield $(23.12 \mathrm{q} / \mathrm{ha})$ which was on par with Soumya (SRCH-55) (22.68 q/ha) and Ajeet 199 (22.39 q/ha). While Super fibre (KDCHB-407) (15.84 q/ha), Surya (Namkot803) (16.77 q/ha), Minerva (SP911B2) (17.33 q/ha) and MRC-7351 (17.86 q/ha) recorded significantly less yield (Table 5).

\section{Grouping of $B t$ cotton genotypes for their reaction to yellow mite}

The data on yellow mite damaged leaf (LCI) were recorded at 15 days intervals starting from 30 DAS. However, the yellow mite population reached its peak at 45 DAS and later gradually decreases up to 90 DAS. Hence data on mean LCI recorded up to 90 DAS have been considered for analysis and interpretation (Table 6).

Grouping of genotypes based on mean leaf curl index indicated that 3 genotypes viz., Chaitanya (MRC-7377), Soumya (SRCH-55) and Ajeet - 199 were resistant; 13 genotypes namely Diamond, Champion, Miracle, Everest, Flux (PCH-888), Gajab, Jadu, MRC-
7383, Ajeet-155, MRC-7353, First class, Bioseed yuva and Nawab were moderately resistant; while, 4 genotypes viz., Minerva (SP911B2), Surya (Namkot-803), Super fibre (KDCHB-407), MRC-7351 were susceptible. While none of the genotypes found to be highly resistant and highly susceptible against yellow mite damage (Table 6).

Screening of selected $B t$ cotton genotypes against yellow mite, Polyphagotarsonemus latus (Banks) during kharif 2018

Out of twenty genotypes screened during kharif 2017, two resistant and two susceptible genotypes were chosen based on morphological characters and leaf curl index and those selected genotypes were grown in kharif 2018 season. The results of the experiment conducted to evaluate the reaction of $4 \mathrm{Bt}$ cotton hybrids against yellow mite along with morphological, biochemical and yield parameters of the genotypes are presented hereunder.

During the 2018 kharif year, four genotypes were screened against cotton yellow mite (Table 7).

The yellow mite population in various $B t$ cotton genotypes was reported at 15 days intervals from 30 DAS to 90 DAS. Chaitanya (MRC-7377) reported the lowest incidence of yellow mite (0.35 yellow mite/ leaf) at 30 days, and the next best genotypes in order were Soumya (SRCH-55) (0.53 yellow mite/ leaf) which recorded significantly less yellow mite population and were on par with each other.

However, more number of yellow mite population recorded on Super fibre (KDCHB407) (3.61 yellow mite/ leaf) followed by Surya (Namkot-803) (3.35 yellow mite/ leaf) and were statistically on par with each other. Furthermore, higher yellow mite population 
registered on super fibre (KDCHB-407) (3.61 yellow mite/ leaf) followed by Surya (Namkot-803) (3.35 yellow mite/ leaf) and was statistically equivalent. The lowest yellow mite population was observed on Chaitanya (MRC-7377) (0.75 yellow mite/ leaf) followed by Soumya (SRCH-55) (0.96 yellow mite/ leaf) which was considerably higher among other genotypes. 60 days after sowing, the trend was close, but the yellow mite population progressively decreased up to 90 days after sowing.

Chaitanya (MRC-7377) recorded the lowest yellow mite population by measuring 0.27 , 0.18 and 0.00 yellow mites per leaf at 60,75 and 90 DAS respectively, followed by Soumya (SRCH-55) (0.33 yellow mite/ leaf, 0.22 yellow mite/ leaf and 0.00 yellow mite/ leaf) and was significantly superior than all genotypes while highest population was recorded in Super fibre as 3.20, 2.57, 0.60 yellow mite per leaf at 60, 75 and 90 DAS respectively.

Moreover, the lowest yellow mite population was estimated in Chaitanya (MRC-7377) (0.31 yellow mite/ leaf), followed by Soumya (SRCH-55) (0.41 yellow mite/ leaf), which was on a par with each other and significantly lower than the remaining genotypes. Super fibre (KDCHB-407) (2.94 yellow mite/ leaf) was recorded the maximum yellow mite population followed by Surya (Namkot-803) (2.78 yellow mite/ leaf) (Table 7).

With respect to leaf curl index, Super fibre (KDCHB-407) registered the highest leaf curl index $(2.39 \mathrm{LCI})$ followed by Surya (Namkot803) (2.30 LCI) which were on par with each other. On the contrary, in Chaitanya (MRC7377) (0.53 LCI) and Soumya (SRCH-55) $(0.58$ LCI $)$ the least leaf curl index was observed, showing significant superiority over other genotypes (Table 7).
Morphological character of selected Bt cotton genotypes for their correlation with mite during kharif 2018

\section{Leaf thickness (mm)}

The leaf thickness presented in table 8 revealed that Chaitanya (MRC-7377) recorded non significantly higher leaf thickness of $0.34 \mathrm{~mm}$ followed by Super fibre (KDCHB-407) (0.29 mm), Surya (Namkot803) $(0.29 \mathrm{~mm})$ and Soumya (SRCH-55) $(0.27 \mathrm{~mm})$ and were on par with each other.

Leaf hair density (No. / $0.5 \mathrm{~cm}^{2}$ )

Significantly higher numbers of leaf hairs were registered in the Chaitanya (MRC-7377) $\left(151.27 / 0.5 \mathrm{~cm}^{2}\right)$ and Soumya (SRCH-55) $\left(131.89 / 0.5 \mathrm{~cm}^{2}\right)$ genotype, while Super fibre (KDCHB-407) $\left(15.18 / 0.5 \mathrm{~cm}^{2}\right)$ and Surya

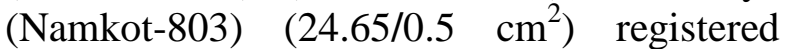
significantly less trichomes. However, the genotypes viz., Flux (PCH-888) (125.50), Gajab (117.44), and Bioseed yuva (112.17) exhibited moderate trichomes (Table 8).

\section{Trichome length (mm)}

The longest trichomes was observed in Chaitanya (MRC-7377) (0.69 mm) and was on par with Soumya (SRCH-55) $(0.64 \mathrm{~mm})$ which were significantly superior while, comparatively smaller trichomes were noticed in Super fibre (KDCHB-407) $(0.14 \mathrm{~mm})$ and Surya (Namkot-803) $(0.17 \mathrm{~mm})$. However, the genotypes viz., Ajeet-199 $(0.40 \mathrm{~mm})$, Gajab $(0.38 \mathrm{~mm})$ and Ajeet-155 $(0.37 \mathrm{~mm})$ exhibited moderate trichome length (Table 8).

Biochemical characters of selected Bt cotton genotypes and their correlation with mite during kharif 2018

The biochemical constituents of leaf act as secondary metabolites/ toxins for many of 
pests and the findings are presented here under.

\section{Total Chlorophyll (mg/g)}

The total chlorophyll content presented in table 9 revealed that Chaitanya (MRC-7377) recorded non significantly higher chlorophyll content of $2.76 \mathrm{mg} / \mathrm{g}$ followed by Super fibre (KDCHB-407) (2.56 mg/g), Surya (Namkot803) $(2.48 \mathrm{mg} / \mathrm{g})$ and Soumya (SRCH-55) $(2.41 \mathrm{mg} / \mathrm{g})$ and were on par with each other.

\section{Sugar (mg/g)}

The biochemical study of total sugar revealed that among $4 \mathrm{Bt}$ cotton genotypes, Chaitanya (MRC-7377) was known to contain significantly low total sugar content $(0.86$ $\mathrm{mg} / \mathrm{g}$ ) followed by Soumya (SRCH-55) (1.12 $\mathrm{mg} / \mathrm{g}$ ) and which were on par with each other. While, Super fibre (KDCHB-407) (3.92 $\mathrm{mg} / \mathrm{g}$ ) and Surya (Namkot-803) (3.13 mg/g) recorded higher total sugar content (Table 9).

\section{Protein $(\mathrm{mg} / \mathrm{g})$}

Estimation of protein among different cotton genotypes revealed that Super fibre (KDCHB407) $(8.27 \mathrm{mg} / \mathrm{g})$ recorded highest protein content followed by Surya (Namkot-803) $(7.62 \mathrm{mg} / \mathrm{g})$ which were on par with each other. The lowest protein content was recorded in Chaitanya (MRC-7377) (3.55 $\mathrm{mg} / \mathrm{g}$ ) and Soumya (SRCH-55) (4.19 mg/g) which were on par with each other (Table 9).

\section{Phenol (mg/g)}

Estimation of phenol revealed that Chaitanya (MRC-7377) (3.55 $\mathrm{mg} / \mathrm{g}) \quad$ recorded significantly higher phenol content (3.39 $\mathrm{mg} / \mathrm{g}$ ) and was on par with Soumya (SRCH55) (3.14 mg/g). However, Super fibre $(\mathrm{KDCHB}-407) \quad(1.21 \mathrm{mg} / \mathrm{g})$ and Surya (Namkot-803) (1.56 mg/g) recorded lower phenol content compared to other genotypes and were on par with each other (Table 9).

\section{Correlation studies}

The correlation coefficient was worked out on the number of yellow mite as a dependent variable and leaf morphological and biochemical characters as independent variables.

The correlation coefficients indicated that there was no significant correlation between the mean number of yellow mite with leaf thickness whereas leaf hair density $(-0.996 * *)$ and trichome length $(-0.999 * *)$ showed highly significant and negative correlation on mean number of yellow mite (Table 8). With respect to biochemical characters, there was a significant negative correlation between numbers of yellow mite with phenol ($0.994 * *)$ whereas positive significant correlation was noticed with Sugars $(0.984 *)$ and protein $\left(0.994^{* *}\right)$ but there was no significant correlation between the mean number of yellow mite with total chlorophyll (Table 9).

Yield and yield parameters of different $B t$ cotton genotypes screened for yellow mite during kharif 2018

\section{Good Opened Bolls (GOB'S)}

Data on GOB's recorded during kharif 2018 revealed that Chaitanya (MRC-7377) (46.39 bolls/ plant) recorded significantly highest number of GOB's followed by Soumya (SRCH-55) (40.28 bolls/ plant.

While, least number of GOB's was recorded in Super fibre (KDCHB-407) (17.97 bolls/ plant) followed by Surya (Namkot-803) (20.41 bolls/ plant) which were significantly on par each other (Table 10). 


\section{Bad Opened Bolls (BOB'S)}

Data on BOB's during kharif 2018 presented in table 15 revealed that Chaitanya (MRC7377) was significantly superior in recording least BOB's ( 8.73 bolls/ plant) and was on par with Soumya (SRCH-55) (11.13 bolls/ plant). While Super fibre (KDCHB-407) (13.18 bolls/ plant) recorded significantly more number of BOB's and was on par with Surya (Namkot-803) (14.62 bolls/ plant) (Table 10).

\section{Seed cotton yield $(q / h a)$}

Data on seed cotton yield during kharif 2018 revealed that Chaitanya (MRC-7377) was significantly superior in recording highest yield $(22.69 \mathrm{q} / \mathrm{ha})$ which was on par with Soumya (SRCH-55) (21.88 q/ha). While Super fibre (KDCHB-407) (16.57 q/ha) and Surya (Namkot-803) (17.16 q/ha) recorded significantly less yield (Table 10).

Table.1 Criteria considered in screening: According to Niles ${ }^{(9)}$ (1980)

\begin{tabular}{|c|l|l|}
\hline $\begin{array}{c}\text { Damage } \\
\text { Score } \\
\mathbf{0}\end{array}$ & Grade & \multicolumn{1}{c|}{ Extent of damage } \\
\hline $\mathbf{1}$ & Grade 0 & No Symptom of leaf curling due to mites \\
\hline $\mathbf{2}$ & Grade 1 & $\begin{array}{l}1-25 \text { per cent of the leaves in plant showing downward curling due to } \\
\text { yellow mite }\end{array}$ \\
\hline $\mathbf{3}$ & $\begin{array}{l}26-50 \text { per cent of the leaves in plant showing downward curling due } \\
\text { to yellow mite (moderately damaged). }\end{array}$ \\
\hline $\mathbf{4}$ & Grade 3 & $\begin{array}{l}51-75 \text { per cent of the leaves in a plant showing downward curling, } \\
\text { heavily damaged, reduction in plant height. }\end{array}$ \\
\hline & Grade 4 & $>75$ per cent of leaves showing curling, severe stunted growth. \\
\hline
\end{tabular}

Table.2 Grouping of genotypes: According to Niles ${ }^{(9)}$ (1980)

\begin{tabular}{|l|c|l|}
\hline \multicolumn{1}{|c|}{ Category } & LCI & \multicolumn{1}{c|}{ Symptom } \\
\hline Highly resistant & $\leq 0.5$ & Completely healthy plant \\
\hline Resistant & $0.51-0.99$ & Healthy plant \\
\hline $\begin{array}{l}\text { Moderately } \\
\text { resistant }\end{array}$ & $1.00-1.99$ & $\begin{array}{l}\text { Few top leaves showing curling (about 25 per cent of } \\
\text { leaves) }\end{array}$ \\
\hline Susceptible & $2.00-2.99$ & $\begin{array}{l}\text { Moderate curling (about 50 per cent of leaves } \\
\text { showing curling) }\end{array}$ \\
\hline Highly susceptible & $\geq 3.00$ & Complete curling (more than 75 per cent showing curling) \\
\hline
\end{tabular}


Table.3 Screening of different Bt cotton genotypes against yellow mite, Polyphagotarsonemus latus during kharif 2017

\begin{tabular}{|c|c|c|c|c|c|c|c|c|c|c|c|c|c|}
\hline \multirow[t]{2}{*}{ Genotype } & \multicolumn{2}{|c|}{ 30 DAS } & \multicolumn{2}{|c|}{ 45 DAS } & \multicolumn{2}{|c|}{ 60 DAS } & \multicolumn{2}{|c|}{75 DAS } & \multicolumn{2}{|c|}{90 DAS } & \multicolumn{2}{|c|}{ Mean } & \multirow[t]{2}{*}{ Cat. } \\
\hline & Mite / leaf & LCI & Mite / leaf & LCI & Mite / leaf & LCI & Mite / leaf & LCI & Mite / leaf & LCI & Mite / leaf & LCI & \\
\hline Ajeet-199 & $\begin{array}{c}0.85 \\
(1.16) \\
\end{array}$ & 0.75 & $\begin{array}{c}1.38 \\
(1.36)\end{array}$ & 0.90 & $\begin{array}{c}0.40 \\
(0.95)\end{array}$ & 0.60 & $\begin{array}{c}0.26 \\
(0.87)\end{array}$ & 0.55 & $\begin{array}{c}0.00 \\
(0.71)\end{array}$ & 0.50 & $\begin{array}{c}0.58 \\
(1.04)^{\mathrm{ab}}\end{array}$ & $0.66^{\mathrm{a}}$ & $\mathbf{R}$ \\
\hline Diamond & $\begin{array}{c}1.78 \\
(1.49)\end{array}$ & 1.65 & $\begin{array}{l}2.28 \\
(1.67)\end{array}$ & 1.800 & $\begin{array}{c}1.74 \\
(1.49)\end{array}$ & 1.65 & $\begin{array}{c}1.21 \\
(1.30)\end{array}$ & 1.60 & $\begin{array}{c}0.28 \\
(0.88)\end{array}$ & 0.55 & $\begin{array}{c}1.46 \\
(1.38)^{\mathrm{e}}\end{array}$ & $1.45^{c}$ & MR \\
\hline Champion & $\begin{array}{c}1.89 \\
(1.54)\end{array}$ & 1.75 & $\begin{array}{c}2.53 \\
(1.74)\end{array}$ & 1.95 & $\begin{array}{r}1.82 \\
(1.51)\end{array}$ & 1.70 & $\begin{array}{c}1.35 \\
(1.35)\end{array}$ & 1.65 & $\begin{array}{c}0.32 \\
(0.90)\end{array}$ & 0.60 & $\begin{array}{c}1.58 \\
(1.44)^{\text {ef }}\end{array}$ & $1.53^{\text {cd }}$ & MR \\
\hline Miracle & $\begin{array}{c}1.1 \\
(1.26)\end{array}$ & 1.20 & $\begin{array}{c}1.82 \\
(1.51)\end{array}$ & 1.55 & $\begin{array}{c}0.91 \\
(1.18)\end{array}$ & 1.10 & $\begin{array}{c}0.66 \\
(1.07)\end{array}$ & 1.10 & $\begin{array}{c}0.15 \\
(0.80)\end{array}$ & 0.50 & $\begin{array}{c}0.93 \\
(1.19)^{\mathrm{c}}\end{array}$ & $1.09^{b}$ & MR \\
\hline Everest & $\begin{array}{c}1.56 \\
(1.43)\end{array}$ & 1.50 & $\begin{array}{c}2.10 \\
(1.62)\end{array}$ & 1.75 & $\begin{array}{l}1.17 \\
(1.28)\end{array}$ & 1.50 & $\begin{array}{c}0.96 \\
(1.18)\end{array}$ & 1.45 & $\begin{array}{c}0.21 \\
(0.84)\end{array}$ & 0.55 & $\begin{array}{c}1.20 \\
(1.30)^{\text {cd }}\end{array}$ & $1.35^{\mathrm{bc}}$ & MR \\
\hline Flux (PCH-888) & $\begin{array}{c}0.91 \\
(1.18)\end{array}$ & 1.05 & $\begin{array}{c}1.44 \\
(1.39)\end{array}$ & 1.30 & $\begin{array}{c}0.77 \\
(1.12)\end{array}$ & 1.05 & $\begin{array}{c}0.45 \\
(0.97)\end{array}$ & 1.00 & $\begin{array}{c}0.00 \\
(0.71)\end{array}$ & 0.50 & $\begin{array}{c}0.71 \\
(1.09)^{b}\end{array}$ & $0.98^{b}$ & MR \\
\hline Gajab & $\begin{array}{c}0.95 \\
(1.19)\end{array}$ & 1.05 & $\begin{array}{r}1.59 \\
(1.44)\end{array}$ & 1.40 & $\begin{array}{c}0.78 \\
(1.12)\end{array}$ & 1.05 & $\begin{array}{c}0.52 \\
(1.03)\end{array}$ & 1.05 & $\begin{array}{c}0.00 \\
(0.71)\end{array}$ & 0.50 & $\begin{array}{c}\mathbf{0 . 7 7} \\
(\mathbf{1 . 1 2})^{\mathrm{b}}\end{array}$ & $1.01^{b}$ & MR \\
\hline Jadu & $\begin{array}{l}1.63 \\
(1.45)\end{array}$ & 1.55 & $\begin{array}{c}2.11 \\
(1.62)\end{array}$ & 1.75 & $\begin{array}{r}1.19 \\
(1.29)\end{array}$ & 1.50 & $\begin{array}{c}1.02 \\
(1.23)\end{array}$ & 1.45 & $\begin{array}{c}0.22 \\
(0.85)\end{array}$ & 0.55 & $\begin{array}{c}1.23 \\
(1.30)^{\mathrm{cd}}\end{array}$ & $1.36^{\mathrm{bc}}$ & MR \\
\hline MRC-7383 & $\begin{array}{c}1.12 \\
(1.27) \\
\end{array}$ & 1.20 & $\begin{array}{c}1.90 \\
(1.54) \\
\end{array}$ & 1.60 & $\begin{array}{c}0.97 \\
(1.21) \\
\end{array}$ & 1.15 & $\begin{array}{c}0.68 \\
(1.07)\end{array}$ & 1.15 & $\begin{array}{c}0.15 \\
(0.80) \\
\end{array}$ & 0.50 & $\begin{array}{c}0.96 \\
(1.18)^{\mathrm{c}}\end{array}$ & $1.12^{b}$ & MR \\
\hline Minerva (SP911B2) & $\begin{array}{c}2.73 \\
(1.79)\end{array}$ & 2.5 & $\begin{array}{c}3.89 \\
(2.08)\end{array}$ & 2.60 & $\begin{array}{c}2.41 \\
(1.68)\end{array}$ & 2.35 & $\begin{array}{c}1.98 \\
(1.57)\end{array}$ & 2.20 & $\begin{array}{c}0.45 \\
(0.97)\end{array}$ & 0.70 & $\begin{array}{c}2.29 \\
(1.67)^{\mathrm{gh}}\end{array}$ & $2.07^{\mathrm{e}}$ & $\mathbf{S}$ \\
\hline Ajeet-155 & $\begin{array}{c}1.00 \\
(1.21)\end{array}$ & 1.10 & $\begin{array}{c}1.80 \\
(1.51)\end{array}$ & 1.55 & $\begin{array}{c}0.90 \\
(1.18)\end{array}$ & 1.10 & $\begin{array}{c}0.62 \\
(1.05)\end{array}$ & 1.10 & $\begin{array}{c}0.12 \\
(0.79)\end{array}$ & 0.50 & $\begin{array}{c}0.89 \\
(1.17)^{\mathrm{bc}}\end{array}$ & $1.07^{\mathrm{b}}$ & MR \\
\hline MRC-7353 & $\begin{array}{l}1.67 \\
(1.47)\end{array}$ & 1.55 & $\begin{array}{c}2.19 \\
(1.63)\end{array}$ & 1.75 & $\begin{array}{l}1.61 \\
(1.44)\end{array}$ & 1.55 & $\begin{array}{r}1.09 \\
(1.25)\end{array}$ & 1.50 & $\begin{array}{c}0.24 \\
(0.86)\end{array}$ & 0.55 & $\begin{array}{c}1.36 \\
(1.36)^{\mathrm{de}}\end{array}$ & $1.38^{\mathrm{bc}}$ & MR \\
\hline $\begin{array}{l}\text { Soumya } \\
\text { (SRCH-55) }\end{array}$ & $\begin{array}{c}0.49 \\
(0.97)\end{array}$ & 0.60 & $\begin{array}{c}0.93 \\
(1.19)\end{array}$ & 0.70 & $\begin{array}{c}0.28 \\
(0.88)\end{array}$ & 0.55 & $\begin{array}{c}0.19 \\
(0.83)\end{array}$ & 0.55 & $\begin{array}{c}0.00 \\
(0.71)\end{array}$ & 0.50 & $\begin{array}{c}0.38 \\
(0.93)^{a}\end{array}$ & $0.58^{\mathrm{a}}$ & $\mathbf{R}$ \\
\hline $\begin{array}{l}\text { Surya } \\
\text { (Namkot-803) }\end{array}$ & $\begin{array}{c}3.28 \\
(1.94)\end{array}$ & 2.80 & $\begin{array}{l}4.62 \\
(2.24)\end{array}$ & 2.90 & $\begin{array}{l}2.85 \\
(1.81)\end{array}$ & 2.60 & $\begin{array}{c}2.54 \\
(1.74)\end{array}$ & 2.45 & $\begin{array}{c}0.55 \\
(1.02)\end{array}$ & 0.75 & $\begin{array}{c}2.77 \\
(\mathbf{1 . 8 1})^{\text {hi }}\end{array}$ & $2.30^{\mathrm{ef}}$ & $\mathbf{S}$ \\
\hline $\begin{array}{l}\text { Super fibre } \\
\text { (KDCHB-407) }\end{array}$ & $\begin{array}{c}3.59 \\
(2.02) \\
\end{array}$ & 2.90 & $\begin{array}{c}4.78 \\
(2.30) \\
\end{array}$ & 2.95 & $\begin{array}{c}3.17 \\
(1.89) \\
\end{array}$ & 2.80 & $\begin{array}{l}2.66 \\
(1.76)\end{array}$ & 2.50 & $\begin{array}{c}0.58 \\
(1.03) \\
\end{array}$ & 0.80 & $\begin{array}{c}2.96 \\
(1.86)^{i}\end{array}$ & $2.39^{\mathrm{ef}}$ & $\mathbf{S}$ \\
\hline $\begin{array}{l}\text { Chaitanya } \\
\text { (MRC-7377) }\end{array}$ & $\begin{array}{c}0.32 \\
(0.90)\end{array}$ & 0.55 & $\begin{array}{c}0.78 \\
(1.12)\end{array}$ & 0.60 & $\begin{array}{c}0.21 \\
(0.84) \\
\end{array}$ & 0.5 & $\begin{array}{c}0.12 \\
(0.79) \\
\end{array}$ & 0.50 & $\begin{array}{c}0.00 \\
(0.71)\end{array}$ & 0.50 & $\begin{array}{c}0.29 \\
(0.88)^{\mathrm{a}}\end{array}$ & $0.53^{\mathrm{a}}$ & $\mathbf{R}$ \\
\hline First class & $\begin{array}{c}1.87 \\
(1.53)\end{array}$ & 1.75 & $\begin{array}{c}2.46 \\
(1.72)\end{array}$ & 1.90 & $\begin{array}{c}1.81 \\
(1.51)\end{array}$ & 1.70 & $\begin{array}{c}1.27 \\
(1.33)\end{array}$ & 1.60 & $\begin{array}{c}0.29 \\
(0.88)\end{array}$ & 0.55 & $\begin{array}{c}1.54 \\
(1.43)^{\text {ef }}\end{array}$ & $1.5 c^{d}$ & MR \\
\hline Bioseed yuva & $\begin{array}{l}1.00 \\
(1.21)\end{array}$ & 1.10 & $\begin{array}{l}1.64 \\
(1.46)\end{array}$ & 1.45 & $\begin{array}{c}0.80 \\
(1.14)\end{array}$ & 1.05 & $\begin{array}{l}0.56 \\
(1.03)\end{array}$ & 1.05 & $\begin{array}{c}0.00 \\
(0.71)\end{array}$ & 0.50 & $\begin{array}{c}0.80 \\
(1.14)^{\mathrm{bc}}\end{array}$ & $1.03^{b}$ & MR \\
\hline Nawab & $\begin{array}{c}1.74 \\
(1.49)\end{array}$ & 1.65 & $\begin{array}{c}2.23 \\
(1.65)\end{array}$ & 1.80 & $\begin{array}{c}1.67 \\
(1.47)\end{array}$ & 1.60 & $\begin{array}{c}1.13 \\
(1.27)\end{array}$ & 1.55 & $\begin{array}{c}0.25 \\
(0.87)\end{array}$ & 0.55 & $\begin{array}{c}1.40 \\
(1.38)^{\text {de }}\end{array}$ & $1.43^{\mathrm{c}}$ & MR \\
\hline MRC-7351 & $\begin{array}{l}2.47 \\
(1.72)\end{array}$ & 2.35 & $\begin{array}{c}3.79 \\
(2.07)\end{array}$ & 2.55 & $\begin{array}{c}2.32 \\
(1.68)\end{array}$ & 2.30 & $\begin{array}{r}1.89 \\
(1.54)\end{array}$ & 2.15 & $\begin{array}{c}0.42 \\
(0.95)\end{array}$ & 0.70 & $\begin{array}{c}2.18 \\
(1.64)^{g}\end{array}$ & $2.01^{e}$ & $\mathbf{S}$ \\
\hline S.Em. \pm & 0.08 & 0.14 & 0.13 & 0.12 & 0.11 & 0.10 & 0.07 & 0.12 & 0.06 & 0.08 & 0.04 & 0.12 & \\
\hline C.D. at 5\% & 0.25 & 0.43 & 0.39 & 0.36 & 0.31 & 0.29 & 0.21 & 0.37 & 0.19 & 0.24 & 0.11 & 0.36 & \\
\hline C.V. (\%) & 9.37 & 11.38 & 7.68 & 9.71 & 5.83 & 10.26 & 10.49 & 9.61 & 6.24 & 7.19 & 5.12 & 8.57 & \\
\hline
\end{tabular}

DAS-Days after sowing, LCI - Leaf Curl Index, Cat.- Category, R - Resistant, MR - Moderately resistant, S - Susceptible

Figures in parentheses are $\sqrt{ } \mathrm{x}+0.5$ transformed values, Means followed by the same letter in a column are not significantly different (P=0.05) by DMRT.

In a column means followed by the same alphabet do not differ significantly by DMRT (0.05) 
Table.4 Morphological character of different Bt cotton genotypes and their correlation with yellow mite during kharif 2017

\begin{tabular}{|c|c|c|c|}
\hline Genotype & Leaf thickness (mm) & Leaf hair density $\left(0.5 \mathrm{~cm}^{2}\right)$ & Trichome length (mm) \\
\hline Ajeet-199 & 0.28 & 131.06 & 0.40 \\
\hline Diamond & 0.32 & 70.02 & 0.28 \\
\hline Champion & 0.33 & 51.83 & 0.29 \\
\hline Miracle & 0.25 & 101.28 & 0.31 \\
\hline Everest & 0.31 & 93.35 & 0.34 \\
\hline Flux (PCH-888) & 0.28 & 125.50 & 0.49 \\
\hline Gajab & 0.31 & 117.44 & 0.38 \\
\hline Jadu & 0.29 & 85.91 & 0.29 \\
\hline MRC-7383 & 0.34 & 95.72 & 0.31 \\
\hline Minerva (SP911B2) & 0.27 & 30.19 & 0.21 \\
\hline Ajeet-155 & 0.36 & 109.61 & 0.37 \\
\hline MRC-7353 & 0.29 & 83.26 & 0.27 \\
\hline Soumya (SRCH-55) & 0.26 & 138.17 & 0.61 \\
\hline Surya (Namkot-803) & 0.30 & 19.63 & 0.19 \\
\hline Super fibre (KDCHB-407) & 0.27 & 12.93 & 0.12 \\
\hline Chaitanya (MRC-7377) & 0.32 & 144.89 & 0.73 \\
\hline First class & 0.30 & 55.21 & 0.30 \\
\hline Bioseed yuva & 0.34 & 112.17 & 0.35 \\
\hline Nawab & 0.26 & 71.68 & 0.27 \\
\hline MRC-7351 & 0.33 & 47.31 & 0.29 \\
\hline S.Em. \pm & - & 0.27 & 0.05 \\
\hline C.D. at $5 \%$ & - & 0.82 & 0.15 \\
\hline C.V. $(\%)$ & NS & 4.18 & 5.63 \\
\hline Correlation of yellow mite with & $-\mathbf{0 . 1 0 3}$ & $-0.974^{* * *}$ & $-0.810^{* * *}$ \\
\hline
\end{tabular}


Table.5 Yield and yield parameters of different $B t$ cotton genotypes screened for yellow mite during kharif 2017

\begin{tabular}{|c|c|c|c|c|}
\hline Genotype & $\begin{array}{l}\text { Bad opened } \\
\text { bolls / plant }\end{array}$ & $\begin{array}{c}\text { Good opened } \\
\text { bolls / plant }\end{array}$ & $\begin{array}{l}\text { Total bolls / } \\
\text { plant }\end{array}$ & $\begin{array}{l}\text { Yield (q/ } \\
\text { ha) }\end{array}$ \\
\hline Ajeet-199 & 7.58 & 44.14 & 51.72 & 22.39 \\
\hline Diamond & 10.73 & 27.64 & 38.37 & 19.12 \\
\hline Champion & 11.24 & 24.68 & 35.92 & 18.17 \\
\hline Miracle & 9.18 & 36.43 & 45.61 & 21.04 \\
\hline Everest & 9.51 & 34.49 & 44.00 & 20.42 \\
\hline Flux (PCH-888) & 7.96 & 42.42 & 50.38 & 21.81 \\
\hline Gajab & 8.22 & 40.67 & 48.89 & 21.49 \\
\hline Jadu & 9.83 & 32.98 & 42.81 & 20.07 \\
\hline MRC-7383 & 9.33 & 35.03 & 44.36 & 20.81 \\
\hline Minerva (SP911B2) & 11.71 & 22.55 & 34.26 & 17.33 \\
\hline Ajeet-155 & 8.93 & 37.80 & 46.73 & 21.18 \\
\hline MRC-7353 & 10.17 & 29.40 & 39.57 & 19.85 \\
\hline Soumya (SRCH-55) & 7.11 & 45.33 & 52.44 & 22.68 \\
\hline Surya (Namkot-803) & 11.89 & 20.54 & 32.43 & 16.77 \\
\hline $\begin{array}{l}\text { Super fibre (KDCHB- } \\
407 \text { ) }\end{array}$ & 12.11 & 17.53 & 29.64 & 15.84 \\
\hline Chaitanya (MRC-7377) & 6.13 & 50.07 & 56.20 & 23.12 \\
\hline First class & 10.97 & 26.64 & 37.61 & 18.78 \\
\hline Bioseed yuva & 8.67 & 39.56 & 48.23 & 21.34 \\
\hline Nawab & 10.41 & 28.71 & 39.12 & 19.53 \\
\hline MRC-7351 & 11.43 & 23.75 & 35.18 & 17.86 \\
\hline S.Em. \pm & 0.23 & 0.17 & 0.19 & 0.22 \\
\hline C.D. at $5 \%$ & 0.69 & 0.52 & 0.57 & 0.65 \\
\hline C.V. (\%) & 5.12 & 5.91 & 5.66 & 9.17 \\
\hline
\end{tabular}

Table.6 Categorization of $B t$ cotton genotypes for their resistance to yellow mite based on morphological characters

\begin{tabular}{|l|c|l|}
\hline \multicolumn{1}{|c|}{ Category } & LCI & \multicolumn{1}{c|}{ Symptom } \\
\hline Highly resistant & $\leq 0.50$ & Nil \\
\hline Resistant & $0.51-$ & Ajeet-199, Soumya (SRCH-55), Chaitanya (MRC-7377) \\
\hline Moderately resistant & 0.99 & \\
& $1.00-$ & Diamond, Champion, Miracle, Everest, Flux (PCH-888), Gajab, \\
& 1.99 & Jadu, MRC-7383, Ajeet-155, MRC-7353, First class, Bioseed yuva, \\
\hline Susceptible & $2.00-$ & Nawab \\
\hline Highly susceptible & 2.99 & M07), MRC-7351 \\
\hline
\end{tabular}


Table.7 Screening of selected Bt cotton genotypes against yellow mite, Polyphagotarsonemus latus (Banks) during kharif 2018

\begin{tabular}{|c|c|c|c|c|c|c|c|c|c|c|c|c|c|}
\hline \multirow[t]{2}{*}{ Genotype } & \multicolumn{2}{|c|}{30 DAS } & \multicolumn{2}{|c|}{45 DAS } & \multicolumn{2}{|c|}{60 DAS } & \multicolumn{2}{|c|}{75 DAS } & \multicolumn{2}{|c|}{90 DAS } & \multicolumn{2}{|c|}{ Mean } & \multirow[t]{2}{*}{ Cat. } \\
\hline & $\begin{array}{c}\text { Mite / } \\
\text { leaf }\end{array}$ & LCI & $\begin{array}{c}\text { Mite / } \\
\text { leaf }\end{array}$ & LCI & $\begin{array}{c}\text { Mite / } \\
\text { leaf }\end{array}$ & LCI & $\begin{array}{c}\text { Mite / } \\
\text { leaf }\end{array}$ & LCI & $\begin{array}{c}\text { Mite / } \\
\text { leaf }\end{array}$ & LCI & $\begin{array}{c}\text { Mite / } \\
\text { leaf }\end{array}$ & LCI & \\
\hline Chaitanya (MRC-7377) & $\begin{array}{c}0.35 \\
(0.92)\end{array}$ & 0.55 & $\begin{array}{c}0.75 \\
(1.12)\end{array}$ & 0.60 & $\begin{array}{c}0.27 \\
(0.88)\end{array}$ & 0.50 & $\begin{array}{c}0.18 \\
(0.82)\end{array}$ & 0.50 & $\begin{array}{c}0.00 \\
(0.71)\end{array}$ & 0.50 & $\begin{array}{c}0.31 \\
(0.89)^{a}\end{array}$ & $\mathbf{0 . 5 3}^{\mathrm{a}}$ & $\mathbf{R}$ \\
\hline Soumya (SRCH-55) & $\begin{array}{c}0.53 \\
(1.01)\end{array}$ & 0.60 & $\begin{array}{c}0.96 \\
(1.21)\end{array}$ & 0.70 & $\begin{array}{c}0.33 \\
(0.90)\end{array}$ & 0.55 & $\begin{array}{c}0.22 \\
(0.84)\end{array}$ & 0.55 & $\begin{array}{c}0.00 \\
(0.71)\end{array}$ & 0.50 & $\begin{array}{c}0.41 \\
(0.95)^{\mathrm{a}}\end{array}$ & $0.58^{\mathrm{a}}$ & $\mathbf{R}$ \\
\hline Surya (Namkot-803) & $\begin{array}{c}3.35 \\
(1.96)\end{array}$ & 2.80 & $\begin{array}{c}4.69 \\
(2.28)\end{array}$ & 2.90 & $\begin{array}{c}2.81 \\
(1.79)\end{array}$ & 2.60 & $\begin{array}{c}2.50 \\
(1.65)\end{array}$ & 2.45 & $\begin{array}{c}0.53 \\
(1.02)\end{array}$ & 0.75 & $\begin{array}{c}2.78 \\
(1.81)^{b}\end{array}$ & $2.30^{b}$ & $\mathbf{S}$ \\
\hline $\begin{array}{l}\text { Super fibre (KDCHB- } \\
\text { 407) }\end{array}$ & $\begin{array}{c}3.61 \\
(2.00)\end{array}$ & 2.90 & $\begin{array}{c}4.74 \\
(2.29)\end{array}$ & 2.95 & $\begin{array}{c}3.20 \\
(1.92)\end{array}$ & 2.80 & $\begin{array}{c}2.57 \\
(1.75)\end{array}$ & 2.50 & $\begin{array}{c}0.60 \\
(1.05)\end{array}$ & 0.80 & $\begin{array}{c}2.94 \\
(1.86)^{b}\end{array}$ & $2.39^{b}$ & $\mathbf{S}$ \\
\hline S.Em. \pm & 0.04 & 0.12 & 0.06 & 0.09 & 0.12 & 0.14 & 0.05 & 0.07 & 0.07 & 0.11 & 0.05 & 0.10 & \\
\hline C.D. at $5 \%$ & 0.13 & 0.37 & 0.18 & 0.27 & 0.35 & 0.40 & 0.15 & 0.22 & 0.21 & 0.32 & 0.16 & 0.31 & \\
\hline C.V. (\%) & 6.73 & 8.68 & 5.94 & 10.42 & 8.19 & 11.57 & 6.38 & 9.71 & 5.23 & 7.84 & 7.46 & 8.26 & \\
\hline
\end{tabular}

DAS-Days after sowing, LCI - Leaf Curl Index, Cat.- Category, R - Resistant, S - Susceptible

Figures in parentheses are $\sqrt{x}+0.5$ transformed values, Means followed by the same letter in a column are not significantly different $(\mathrm{P}=0.05)$ by $\mathrm{DMRT}$ In a column means followed by the same alphabet do not differ significantly by DMRT (0.05)

Table.8 Morphological characters of selected Bt cotton genotypes and their correlation with yellow mite during kharif 2018

\begin{tabular}{|c|c|c|c|}
\hline Genotype & Leaf thickness (mm) & Leaf hair density $\left(0.5 \mathrm{~cm}^{2}\right)$ & Trichome length (mm) \\
\hline Chaitanya (MRC-7377) & 0.34 & 151.27 & 0.69 \\
\hline Soumya (SRCH-55) & 0.27 & 131.89 & 0.64 \\
\hline Surya (Namkot-803) & 0.29 & 24.65 & 0.17 \\
\hline S.Em. \pm & - & 0.22 & 0.04 \\
\hline C.D. at $5 \%$ & - & 0.65 & 0.12 \\
\hline C.V. $(\%)$ & NS & 6.73 & 4.82 \\
\hline
\end{tabular}

**. Correlation is significant at the 0.01 level (2-tailed).

*. Correlation is significant at the 0.05 level (2-tailed). 
Table.9 Biochemical characters of selected Bt cotton genotypes and their correlation with yellow mite during kharif 2018

\begin{tabular}{|c|c|c|c|c|}
\hline Genotype & Total chlorophyll (mg / g) & Sugars (mg / g) & Protein (mg / g) & Phenol (mg / g) \\
\hline Chaitanya (MRC-7377) & 2.76 & 0.86 & 3.55 & 3.39 \\
\hline Surya (Namkot-803) & 2.48 & 3.13 & 7.62 & 1.56 \\
\hline S.Em. \pm & - & 0.04 & 0.05 & 0.07 \\
\hline C.D. at 5\% & - & 0.13 & 0.15 & 0.20 \\
\hline C.V. $(\%)$ & NS & 1.65 & 2.14 & 3.19 \\
\hline
\end{tabular}

Table.10 Yield and yield parameters of different Bt cotton genotypes screened for yellow mite during kharif 2018

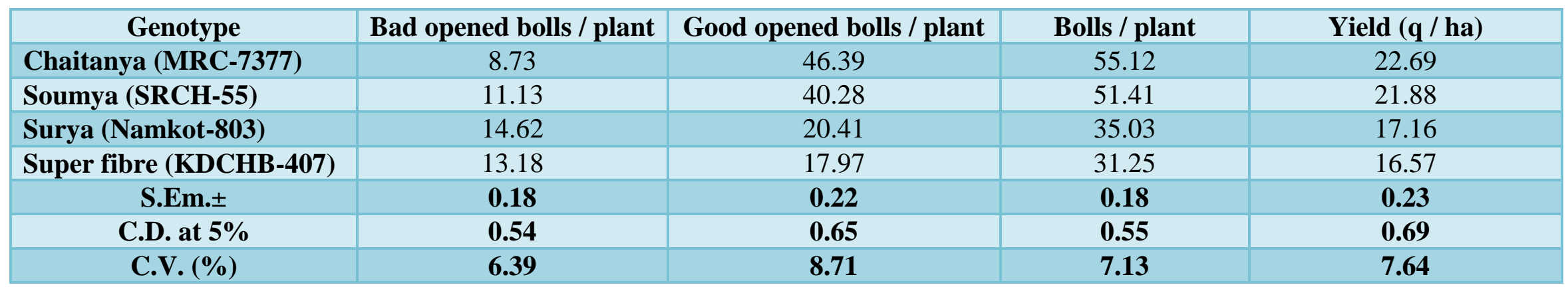


During kharif 2017, among the 20 genotypes screened indicated that lowest mean population of yellow mite was recorded in Chaitanya (MRC-7377) (0.29 yellow mite/ leaf) followed by Soumya (SRCH-55) and were superior to all the genotypes. While highest mean population was observed in Super fibre (KDCHB-407) (2.96 yellow mite/ leaf) followed by Surya (Namkot-803) and were found inferior to all other genotypes. Super fibre (KDCHB-407) recorded the highest leaf curl index (2.39 LCI) which was on par with Surya (Namkot-803). Least leaf curl index was observed in Chaitanya (MRC7377) $(0.53$ LCI $)$ followed by Soumya (SRCH-55) and Ajeet - 199.

Among 20 genotypes observed, Ajeet - 155 recorded more leaf thickness followed by Bioseed yuva, MRC - 7383 and Champion. With respect to hair density, genotype Chaitanya (MRC-7377), Soumya (SRCH-55) and Ajeet - 199 were recorded highest trichomes while Super fibre (KDCHB-407) and Surya (Namkot-803) had least trichomes. However, the genotypes viz., Flux (PCH-888), Gajab, Bioseed yuva and Ajeet - 155 had moderate leaf hair density. Similarly longer trichomes was observed in in Chaitanya (MRC-7377) and was on par with Soumya (SRCH-55) and Flux (PCH-888) which were significantly superior to all the genotypes while smaller trichomes were noticed in fibre (KDCHB-407), Surya (Namkot-803) and Minerva (SP911B2).

With respect to yield parameters, Chaitanya (MRC-7377) was significantly superior in recording least BOB`s, highest GOB`s and yield followed by Soumya (SRCH-55) and Ajeet - 199. While, highest BOB`s, least GOB`s and yield were recorded in Super fibre (KDCHB-407), Surya (Namkot-803), Minerva (SP911B2).

Grouping of genotypes against yellow mite damage indicated that 3 genotypes viz.,
Chaitanya (MRC-7377), Soumya (SRCH-55) and Ajeet - 199 were resistant; 13 genotypes namely Diamond, Champion, Miracle, Everest, Flux (PCH-888), Gajab, Jadu, MRC7383, Ajeet-155, MRC-7353, First class, Bioseed yuva and Nawab were moderately resistant; while, 4 genotypes viz., Minerva (SP911B2), Surya (Namkot-803), Super fibre (KDCHB-407), MRC-7351 were susceptible. While none of the genotypes found to be highly resistant and highly susceptible against yellow mite damage.

For biochemical character analysis during kharif 2018, two resistant and two susceptible genotypes were chosen based on performance during kharif 2017. Morphological characters like trichomes and trichome length and biochemical character phenol were recorded highest in resistant genotypes Chaitanya (MRC-7377) and Soumya (SRCH-55). While, susceptible genotypes Super fibre (KDCHB407) and Surya (Namkot-803) were recorded lowest trichomes and trichome length and highest sugars and proteins. However, leaf thickness and total chlorophyll were did not vary the infestation of yellow mite.

The correlation coefficients indicated that there was a significant negative correlation between numbers of yellow mite with leaf hair density, trichome length and phenol whereas leaf thickness and total chlorophyll shows negative non significant relationship. Similarly, a positive significant correlation was noticed with sugars and protein.

The present findings of different characters are in conformity with Ramya P R ${ }^{(10)}$ (2012), Uppar $^{(11)}$ (2014), Harish ${ }^{(4)}$ (2015) and Girish et al., ${ }^{(3)}$ (2018).

In conclusion, the yellow mite incidence was no significant correlation with leaf thickness while leaf hair density and trichome length showed highly significant and negative correlation. With respect to biochemical 
characters, there was no significant correlation between the mean number of yellow mite with total chlorophyll but significant negative correlation between numbers of yellow mite with phenol whereas positive significant correlation was noticed with sugars and protein.

\section{Acknowledgement}

Authors gratefully acknowledge the support given by the College of Agriculture, Hanumanamatti and College of Agriculture, Dharwad for providing material and conducting study.

\section{References}

Anonymous 2016. Cotton Industry India. https://www.ibef.org/exports/cottonindustry india.aspx, pp. 85.

Butter NS, Vir BK, Kaur G, Singh TH, Raheja RK. Biochemical basis of resistance to whitefly Bemisia tabaci Genn. (Aleyrodidae: Homoptera) in cotton. Tropical Agriculture. 1992; 69: 119-122.

Girish R, Srinivasa N, Basanth YS, Shruthi HR. Response of chilli genotypes to yellow mite, Polyphagotarsonemus latus Banks population and biochemical basis of resistance. Journal of Entomology and Zoology Studies. 2019; 7(1): 250-255.

Harish KMB. Dynamics and management of sucking pest complex in $B t$ cotton. M.Sc (Agri) Thesis, University of Agricultural Sciences. 2015; Dharwad.
Hiscox JD, Israelstam GF. A method of extraction of chlorophyll from leaf tissue without maceration. Canadian journal of Botany. 1979; 57: 1332-1334.

Jeya PS, Regupathy A. Generating base line data for insecticide resistance monitoring in cotton leafhopper, Amrasca devastans (Distant). Resistace Pest Managementt Newsletter. 2002; 11(2): 4-5.

Lowry Oliver H, Nira J, Rosebrough A, Lewis Farr, Rose J Randall. 1951, Protein measurement with the folin phenol reagent. Journal Biological Chemistry. $1951 ; 193 ; 265-275$.

Nelson N. A photometric adaptation of Somogyi method for determination of glucose. Journal Biological Chemistry. 1944; 153: 375-378.

Niles GA. Breeding cotton for resistance to insect pests. In Breeding plant resistance to insects. Macwell. FG. Jennings (Eds) PR John Wiley and Sons, NewYork, 1980 : pp. 337-369.

Ramya PR. Bio-ecology and management of yellow mite, Polyphagotarsonemus latus (banks) on Bt cotton. M.Sc. (Agri) Thesis, University of Agricultural Sciences. 2012; Raichur.

Sadasivam S, Manickam A. Biochemical Methods for Agricultural Sciences. Wiley Eastern Ltd., New Delhi. 1992; pp- 242.

Uppar VV. Evaluation of different management strategies against thrips in $B t$ cotton. Ph. D Thesis, University of Agricultural Sciences. 2014; Dharwad.

\section{How to cite this article:}

Biradarpatil, K. A., S. T. Prabhu and Ravikumar, M. R. 2020. Screening of Different Bt Cotton Genotypes against Yellow Mite, Polyphagotarsonemus latus (Banks) and Biochemical Basis of Resistance. Int.J.Curr.Microbiol.App.Sci. 9(01): 1555-1569. doi: https://doi.org/10.20546/ijcmas.2020.901.173 